\title{
Los cambios en la productividad del sector bancario colombiano en el período 2002-2016
}

Gloria Rodríguez-Lozano*

Fecha de recepción: 3 de junio de 2020

Fecha de aceptación: 1 de diciembre de 2020

Resumen: En este artículo se estudia la evolución de la productividad de los bancos en Colombia desde el año 2002 hasta el 2016, mediante la aplicación de la metodología no paramétrica del índice de Malmquist de Data Envelopment Analysis (DEA), a fin de generar indicadores de eficiencia relativa para cada uno de los bancos por cada periodo, a lo largo de los quince años del estudio. Los resultados muestran que en el $64 \%$ de los periodos la productividad promedio mejoró, siendo el lapso 2003-2004 el que tuvo el mejor resultado, pues se considera que durante ese tiempo el sector bancario colombiano estuvo en pleno desarrollo y consolidación. Adicionalmente, en cuatro periodos hubo disminución de la productividad promedio, la mayor disminución ocurrió entre 2009-2010, durante la primera crisis financiera mundial de este siglo.

Palabras clave: índice de Malmquist; medición de la productividad; DEA; sector bancario; política monetaria; recursos financieros.

Clasificación JEL: G21, C69, C02, G14, G20.

Cómo citar este artículo:

Rodriguez Lozano, G. (2021). Los cambios en la productividad del sector bancario colombiano en el período 2002-2016. Apuntes del Cenes, 40(71). Págs. 105 - 132. https://doi.org/10.19053/01203053.v40.n71.2021.11120

\footnotetext{
* Doctora en Ciencias Económicas. M. C. en Ingeniería Industrial. Administradora de Empresas. Profesora asociada de la Facultad de Ciencias Económicas de la Universidad Nacional de Colombia. girodriguezl@unal.edu.co (iD http://orcid.org/0000-0001-6906-9223
} 


\title{
Changes in Productivity of the Colombian Banking Sector during 2002-2016
}

\begin{abstract}
In this paper the evolution of the productivity of banks in Colombia from 2002 to 2016 is studied through the application of the non-parametric methodology of the Malmquist index of Data Envelopment Analysis (DEA), generating relative efficiency indicators for the banks for each period, throughout the fifteen years of the study. The results show that in $64 \%$ of the periods the average productivity improved, and the period 2003-2004 had the best result, since during this time the Colombian banking sector was in full development and consolidation. Additionally, in four periods there was a decrease in average productivity, the greatest decrease occurred between 2009 and 2010, during the first world financial crisis of this century.
\end{abstract}

Keywords: Malmquist index; productivity measurement; DEA; banking sector; monetary policy; financial resources. 


\section{INTRODUCCIÓN}

El sector bancario es de gran importancia en todo el mundo, pues es el llamado a realizar la asignación y provisión de recursos monetarios; actividad fundamental que tiene un impacto directo en el progreso de los países. Tanto es así que para Berger et al. (2004) los mercados financieros tienen un poderoso efecto sobre el desarrollo económico tanto regional como individual de cada país. Al vincular inversores y ahorradores, los bancos desempeñan un papel crucial en la asignación de recursos, pues diversifican los riesgos y reducen las desigualdades de la información en los mercados crediticios. De ahí que el acceso limitado a préstamos para particulares y empresas dificulte la inversión en capital humano y físico, la innovación y el crecimiento de la productividad. Además del crecimiento a largo plazo, la evidencia empírica también muestra que el acceso a la financiación cumple una función importante en la conformación de las disparidades económicas entre individuos (Cihák et al., 2012). Las restricciones al acceso financiero reducen las oportunidades económicas para quienes carecen de riqueza, condición social y conexiones políticas. Por otra parte, un sistema financiero que funcione bien, reduce la brecha entre las partes inferiores y superiores de la distribución del ingreso en las economías emergentes y en desarrollo (Beck et al., 2007). Aún más, en los países con economías de mercado emergentes, como Colombia, y que cuentan con un sector bancario importante, se presenta recurrentemente un mayor nivel de desarrollo económico que aquellas que tienen un sector bancario más limitado (IDB, 2004). Según Singh et al. (2005), en la mayoría de estos países los bancos comerciales son los que ejecutan prioritariamente la intermediación financiera. Por eso, para el IDB (2004) los bancos "Tienen la capacidad de estimular y recolectar el ahorro de una sociedad y distribuirlo entre las empresas y los sectores que necesitan capital como insumo para sus actividades económicas". Pero también es cierto que en América Latina el acceso a los servicios financieros en general, y al crédito en particular, es bajo por la ineficiencia que se presenta en la región (García et al., 2013). 
Dentro del sistema financiero colombiano, los bancos hacen parte de los establecimientos de crédito (Uribe, 2013) supervisados por la Superintendencia Financiera de Colombia (SFC), junto con las compañías de financiamiento tradicional, las compañías de financiamiento especializadas en leasing, las cooperativas financieras y las corporaciones financieras. Para Coltefinanciera (2017), los establecimientos de crédito son instituciones financieras que tienen como función más importante "captar en moneda legal recursos del público, ya sea en depósitos a la vista (cuentas de ahorro, corriente) o a término (CDT y CDAT), para colocarlos nuevamente a través de préstamos, descuentos, anticipos $\mathrm{u}$ otras operaciones activas de crédito". Ser vigilado por la SFC significa "que existe una institución que autoriza y vigila la actividad que realizan las entidades que reciben dineros del público, donde usted ahorra, invierte su capital, tiene un crédito, un seguro o su pensión" (SFC, 2017). Según el artículo 2 del Estatuto Orgánico del Sistema Financiero, (Decreto 663 de abril 2 de 1993), la principal función de los establecimientos bancarios es "la captación de recursos en cuenta corriente bancaria, así como también la captación de otros depósitos a la vista o a término, con el objeto primordial de realizar operaciones activas de crédito" (Secretaría del Senado, 2017).

Dada la importancia del sector bancario en el mundo, en este artículo se analiza su comportamiento durante los últimos
15 años, utilizando la metodología no paramétrica del índice de Malmquist de Data Envelopment Analysis (DEA), para generar indicadores de eficiencia relativa para cada uno de los bancos y por cada periodo, desde el año 2002 y hasta el año 2016. Y se responden las siguientes preguntas: ¿Cuáles son los índices de Malmquist (IM) para cada banco en cada periodo de estudio? ¿Cómo es el comportamiento del IM promedio? ¿Cómo impactan las adquisiciones entre bancos al IM? ¿Cómo es el comportamiento del IM de los diferentes grupos dentro del sector bancario colombiano?

\section{EL SECTOR BANCARIO EN COLOMBIA}

El Banco de la República fue creado en 1923 como la más importante autoridad monetaria, cambiaria y crediticia en Colombia, con el principal objetivo de asegurar la estabilidad del nivel de precios y de las políticas macroeconómicas del país. Es responsable de la emisión de moneda, asume el papel de banquero de los bancos, diseña y decide respecto al tratamiento de la política del tipo de cambio y se encarga de la administración de las reservas nacionales. Con el propósito de permitir el funcionamiento estable del sector financiero, en el año de 1923 se expidió la Ley 25, que estableció el Estatuto Orgánico del Banco de la República, y la Ley 45, que reglamentó los bancos privados y la Superintendencia Bancaria (Meisel, 1990). 
Más adelante y con el objeto de dar una mayor estabilidad al sector, se creó el Fondo de Garantías de Instituciones Financieras (Fogafín) mediante la Ley 117 de 1985, para proteger la confianza de los depositantes y acreedores de las entidades financieras registradas, preservar el equilibrio económico y la igualdad, y evitar la obtención de beneficios injustificados por parte de los accionistas y administradores de las instituciones financieras que puedan atentar contra la estabilidad del sistema financiero.

Fogafín hace parte de la Red de Seguridad del Sistema Financiero colombiano, conformada por el Ministerio de Hacienda y Crédito Público, el Banco de la República y la Superintendencia Financiera de Colombia (Fogafín, 2017). En la década de los noventa se inicia la transición hacia un sistema de banca universal, con énfasis en los bancos comerciales. Se normatizó un esquema de filiales y matrices, se redefinió la estructura del sector y sus servicios, y se liberalizó la entrada y salida de los mercados (Arango, 2006).

En las dos últimas décadas, el sistema bancario colombiano se ha abierto más a las inversiones privadas y extranjeras. A principios de los años noventa, el sistema se caracterizó por un alto nivel de participación estatal y un nivel de contestabilidad relativamente bajo (Cuartas et al., 2012). La contestabilidad mide el grado en que las barreras de entrada y salida restringen las nuevas entradas, de manera que los mercados contestables tienen barreras limitadas. Colombia comenzó a abrir su sistema financiero a inversionistas extranjeros y la participación estatal en actividades bancarias directas fue sustancialmente reducida. Por ejemplo, mientras que en 1991 los bancos estatales representaban más de la mitad de los activos totales del sistema, ya en 1996 esta proporción se había reducido aproximadamente al $20 \%$. Como consecuencia, la competencia se incrementó sustancialmente (Barajas et al., 1999). La mayor contestabilidad y más participación extranjera y privada en la década de 1990 llevó a un aumento en la profundidad financiera (Daude \& Pascal, 2015). Sin embargo, la crisis bancaria de 1998-2000 fue en parte una consecuencia de este crédito de expansión rápida, ya que la calidad del préstamo disminuyó significativamente, los aumentos de las tasas de interés para defender el tipo de cambio presionaron al servicio de la deuda y los desequilibrios monetarios terminaron causando problemas de solvencia una vez el tipo de cambio fue devaluado (Gómez-González \& Kiefer, 2007).

En la siguiente década, además de construir un fuerte marco de regulación y supervisión bancaria, se profundizó el proceso de reducción de las barreras a la inversión extranjera en el sector. Para el año 2015, la participación de los bancos de propiedad extranjera es de alrededor del $25 \%$ del total de 
activos, mientras que el Banco Agrario es el único banco del Gobierno con una participación del mercado de aproximadamente solo el $5 \%$.

La concentración en el sector bancario colombiano ha seguido una tendencia al alza. El proceso de consolidación derivado de la crisis de 1998-2000 se profundizó en la siguiente década (Daude \& Pascal, 2015). En los últimos años, el sector bancario colombiano está siendo dominado por unos cuantos grandes conglomerados con no más de diez instituciones financieras que poseen casi el $86 \%$ de los activos totales del sector financiero y los tres principales bancos, que representan aproximadamente el $50 \%$ del total de activos y el total de préstamos brutos en 2013. Mientras que la SFC registró la creación de nueve nuevos bancos entre 2008 y 2013, solo representan menos del $3 \%$ del mercado. En general, el sector bancario colombiano tiene una mayor concentración, pero para el año 2013 es moderada según estándares internacionales, pues es similar a la concentración promedio en Europa según cálculos de la OCDE. Pero adicionalmente, una propiedad más concentrada puede inducir un mejor monitoreo y un efecto positivo en la rentabilidad y la productividad (Claessens \& Djankov, 1999).

Para el año 2016 en Colombia hay cuatro importantes grupos financieros nacionales con operaciones internacionales: Grupo Aval, Grupo Bancolombia,
Grupo Bolívar y el Grupo Colpatria. El Grupo Aval es uno de los grupos financieros más importantes de Colombia, incluso en su página web se autodenomina como "El grupo financiero más grande de Colombia", específicamente (Grupo Aval, 2017): es el conglomerado financiero más grande de Colombia y a través de BAC Credomatic es el grupo regional más grande y más rentable en Centroamérica. Cuenta con cuatro bancos: Banco de Bogotá, Banco de Occidente, Banco Popular y Banco AV Villas. Banco de Bogotá: es la institución financiera de mayor trayectoria en el país (fundado en 1870), el segundo Banco más grande de Colombia por tamaño de activos y la compañía más grande del Grupo Aval por su nivel de activos, depósitos y utilidad. Banco de Occidente: es el quinto banco más grande de Colombia por nivel de activos y cartera y el tercer banco más grande en cuentas corrientes. Banco Popular: es pionero en el mercado de créditos de libranza y provee soluciones financieras para entidades gubernamentales en Colombia. Banco AV Villas: ha pasado de estar enfocado exclusivamente en los préstamos de vivienda a ser un banco universal orientado al consumo, es el banco más activo del grupo en el uso de canales no tradicionales: banca móvil, corresponsales no bancarios y sucursales virtuales.

Otro de los importantes grupos es el Grupo Bancolombia: indica que han pasado 142 años desde su nacimiento 
(Grupo Bancolombia, 2017); este grupo se origina por la fusión del Banco de Colombia y del Banco Industrial Colombiano, posteriormente se fusionó con Conavi (2005) y Confisura. Bancolombia es el banco privado más grande del país por el tamaño de su patrimonio y de sus activos (Grupo Sura, 2017).

El Grupo Empresarial Bolívar es un conglomerado de empresas que están coordinadas y controladas a través de Sociedades Bolívar. A este grupo pertenece el Banco Davivienda que tuvo su origen en la Corporación Colombiana de Ahorro y Vivienda, Coldeahorro (fundada en 1972) y los Bancos Superior (fusión en el 2005) y el Granbanco-Bancafé (Davivienda, 2017). Según la información de su página web, "Actualmente se encuentra consistentemente posicionado entre los tres primeros bancos del país", con un número récord de clientes para el año 2016 de 7714552 y 593 oficinas (Banco Davivienda, 2017).

El Grupo Colpatria-Red Multibanca tiene unidad bancaria (banco y fiduciaria), unidad constructora, unidad de inversión y a Colfondos. En octubre del 2011, Colpatria, holding del grupo, anunció el nuevo socio del Banco: la multinacional Scotiabank, con quien firmaron una alianza estratégica a largo plazo; esta multinacional es una de las principales instituciones financieras de Norteamérica y de Canadá (Colpatria, 2017).
Los grupos financieros internacionales con presencia en Colombia son: BBVA, Citibank, GNB Sudameris y Corpbanca. Grupo BBVA: compuesto por una entidad bancaria española con más de un siglo y medio de trayectoria, que después de fusiones y adquisiciones tanto nacionales como internacionales, en la actualidad es el Grupo BBVA. En 1996 llegó a Colombia mediante la compra del $40 \%$ del Banco Ganadero, en el año 2004 pasó a llamarse BBVA Colombia y en 2006 se fusiona con el Banco Granahorrar (BBVA, 2017).

Grupo Citibank: la historia de Citibank en el mundo se inicia en Estados Unidos en 1812, cuando se funda el City Bank of New York (hoy Citibank), (Citibank, 2017). Citibank Colombia es una franquicia de Citigroup. El grupo está compuesto por la sociedad controlante, Citibank Colombia S.A. y las subordinadas (Citibank Colombia, 2017). Citi en Colombia abrió la primera sucursal en 1916, desde 1986 se organizó según el modelo de Banca de Consumo y Banca Corporativa (Citigroup, 2017).

Grupo GNB Sudameris: en 1920 nace el banco como una sociedad mercantil colombiana, después de adquisiciones y ventas en 2004 Banco Sudameris adquiere la mayoría accionaria del Banco Tequendama y de Servibanca. A inicios de 2014 se formaliza la incorporación de la operación de HSBC. Para 2017 el grupo se compone del Banco GNB Sudameris y siete empresas (Banco GNB Sudameris, 2017). 
Grupo Corpbanca: el Banco Corpbanca se generó en el año 1997 de la unión del Banco Concepción Chileno y el Banco Corp Group. Desde entonces se posicionó en el mercado chileno como el cuarto banco más importante. En el año 2012 llegó al mercado colombiano (Corpbanca, 2015). En 2017 el Banco Corpbanca Colombia contaba con cuatro empresas filiales.

Ahora bien, al corte de diciembre de 2016 y teniendo como fuente de información a la SFC, se observa que dentro del sistema financiero colombiano hay 25 bancos que representan el $11 \%$ del total de las entidades del sector; pero, en términos de participación en activos, los bancos representan el $93 \%$. Mientras que, por ejemplo, las cooperativas de ahorro y vivienda tienen una participación del $78 \%$ en las entidades, pero solo el $2.1 \%$ de participación en los activos del sector. Dentro de los mencionados 25 bancos hay 14 nacionales (incluidos los dos de propiedad cooperativa), diez extranjeros y uno público.

El sector bancario cerró el año 2016 con un total de activos de $\$ 548$ billones con un incremento del $8.6 \%$ respecto al año anterior y del $126 \%$ en relación con el resultado para el año 2010, teniendo en este periodo una tendencia creciente (Asobancaria, 2017); las entidades con mayores activos al cierre de 2016 fueron el Bancolombia, el Banco de Bogotá y Davivienda.
Respecto a la cartera bruta, 2016 cerró con $\$ 394$ billones que representa un incremento del $12 \%$ con relación a 2015 y de $148 \%$ respecto al año 2010 , con una tendencia siempre creciente. Para el cierre 2016, la cartera comercial del sector bancario participa con el $58 \%$, y la de consumo con el $27 \%$, vivienda con el $13 \%$ y la de microcrédito apenas con el $3 \%$; estas participaciones son muy similares a las del año inmediatamente anterior (Asobancaria, 2017).

Mirando el comportamiento del pasivo se establece que al cierre de 2016 es de 475 billones con un crecimiento para este último año del $9 \%$, y entre los años 2010 y 2016 con un crecimiento del $125 \%$ con tendencia siempre creciente. La relación entre la cartera otorgada y los depósitos del público (ahorro, CDT y cuentas corrientes) para el sector es de 0,92 lo que representa que el sector por cada peso que capta coloca solo 0,92 pesos.

\section{MATERIALES Y MÉTODOS}

\section{Data Envelopment Analysis (DEA)}

Es una metodología de programación lineal avanzada no paramétrica, con un doble proceso de optimización, la cual establece la eficiencia relativa de unidades organizacionales que toman decisiones (Decision Making Unit -DMU). Yang et al. (2014) explican que esto se realiza mediante la generación de una frontera eficiente que sitúa los índices relativos individuales sin haber 
establecido previamente la función de producción. De manera más específica, DEA se aplica para evaluar la eficiencia relativa de un conjunto de $n$ DMU, por medio del planteamiento de problemas de programación lineal para cada unidad de acuerdo con los datos de utilización de recursos o entradas y generación de productos o salidas (Wei \& Yang, 2010). De manera más amplia, Dotoi et al. (2015) consideran que la eficiencia de cada DMU se define como la relación entre las salidas ponderadas y las entradas ponderadas, de tal manera que se obtiene mediante la solución de un problema doble de programación lineal con el fin de determinar el óptimo conjunto de pesos que maximiza.

DEA ha experimentado un desarrollo dinámico, convirtiéndose gradualmente en un conjunto de conceptos y metodologías, los cuales se han concretado en una serie de modelos (Rodríguez, 2011). El primer modelo DEA en ser desarrollado fue el modelo CRS (Constant Returns to Scale) en 1978, que da como resultado la clasificación categórica de cada DMU (Iyer \& Banerjee, 2016); luego de algunos años aparece el modelo VRS (Variable Returns to Scale) mediante el cual se pueden trabajar retornos no solo constantes (Ghasemi et al., 2015), pues Lau (2013) aclara que estos retornos también pueden ser incrementales y decrementales.

\section{Índice de productividad de Malmquist}

El índice de productividad de Malmquist es otro de los modelos DEA desarrollados, introducido en la literatura por Caves et al. (1982). El índice cuantifica el cambio en la productividad multifactor experimentado por la empresa durante el período de tiempo medido por la relación de la función de distancia de Shephard (Shepard, 1970), evaluado en los niveles reales de input-output de una empresa en dos diferentes puntos en el tiempo.

Una década más tarde, Fare et al. (1992) mostraron cómo se podía utilizar la programación matemática para evaluar las funciones de distancia cuya relación mide el índice de Malmquist. El desarrollo de Caves et al. (1982) asumió que la tecnología mostraba rendimientos constantes a escala (CRS) y también que los conjuntos de entrada-salida observados eran técnicamente eficientes. Aunque Fare et al. (1992) mantuvieron el supuesto de CRS, el desarrollo permitió ineficiencia técnica. Dentro de este marco analítico, identificaron el cambio tecnológico o también denominado "Boundary Shift" y el cambio de eficiencia relativa también llamado "Efficiency Catch", como dos componentes distintos del cambio de productividad.

Posteriormente, Fare et al. (1994) relajaron la hipótesis de CRS permitiendo rendimientos variables a escala (VRS) $\mathrm{y}$ ofrecieron una nueva descomposi- 
ción del índice de productividad de Malmquist, incorporando un nuevo componente que representa la contribución de los cambios en la eficiencia de la escala. Al comentar esta composición, Ray y Desli (1997) cuestionaron la validez de usar las relaciones de las funciones de distancia CRS para medir variables que serían relevantes solo cuando la tecnología tiene VRS. De tal manera que Ray y Desli (1997) plantearon una descomposición alternativa que mide apropiadamente el cambio tecnológico por la relación de las funciones de distancia de la VRS.

Ahora bien, Grifell-Tatjé y Lovell (1999) platean matemáticamente de la siguiente manera:

Sea $x^{t} \in R^{N_{+}}$y $y^{t} \in R^{M_{+}}$un vector de entrada y un vector de salida de un productor en el periodo $t, t=1, \ldots, T$. El conjunto de producción

$$
S^{t}=\left\{\left(y^{t}, x^{t}\right): x^{t} \text { puede producir } y^{t}\right\}
$$

es el conjunto de todos los vectores viables de entrada de salida en el periodo t. Los conjuntos de salida asociados con $\mathrm{St}$,

$$
P^{t}\left(x^{t}\right)=\left\{y^{t}:\left(y^{t}, x^{t}\right) \in S^{t}\right\}, x t \in R^{N_{+}}
$$

Se asume que son cerrados, limitados, convexos, y que satisfacen la fuerte disponibilidad de salidas e insumos. No se impone ninguna restricción sobre la naturaleza de los rendimientos de escala. Una representación funcional de la estructura de la tecnología es proporcionada por la función de distancia de salida de Shephard

$$
D_{0}{ }^{t}\left(x^{t}, y^{t}\right)=\min \left\{\theta:\left(y^{t} / \theta\right) \in P^{t}\left(x^{t}\right)\right\}
$$

$D_{0}{ }^{t}\left(x^{t}, y^{t}\right) \leq 1, \quad$ con $\quad D_{0}{ }^{t}\left(x^{t}, y^{t}\right)=1 \Leftrightarrow y^{t} \in$ Isog $\mathrm{P}^{\mathrm{t}}\left(\mathrm{x}^{\mathrm{t}}\right)=\left\{\mathrm{y}^{\mathrm{t}}: \mathrm{y}^{\mathrm{t}} \in \mathrm{P}^{\mathrm{t}}\left(\mathrm{x}^{\mathrm{t}}\right), \lambda \mathrm{y}^{\mathrm{t}} \notin \mathrm{P}^{\mathrm{t}}\left(\mathrm{x}^{\mathrm{t}}\right), \lambda>1\right\}$.

Además, $D_{o}^{t}\left(x^{t}, \lambda y^{t}\right)=\lambda D_{0}^{t}\left(x^{t}, y^{t}\right), \lambda>0$.

Finalmente, $D_{0}{ }^{t}\left(x^{t}, y^{t}\right)$ es una función de distancia de salida dentro del período.

Las funciones de distancia de salida del período adyacente se definen como

$$
D_{0} t\left(x^{t}, y^{t}\right)=\min \left\{\theta:\left(y^{t+1} / \theta\right) \in P^{t}\left(x^{t+1}\right)\right\}
$$

Y

$$
\operatorname{Do}^{t+1}\left(x^{t}, y^{t}\right)=\min \left\{\theta:\left(y^{t} / \theta\right) \in P^{t+1}\left(x^{t}\right)\right\}
$$

respectivamente.

Dado que los datos de un período pueden no ser factibles con la tecnología durante el periodo adyacente,

$D_{0} t\left(x^{t+1}, y^{t+1}\right) \geq \leq 1 y^{t+1}\left(x^{t}, y^{t}\right) \geq \leq 1$

Para Caves et al. (1982), un índice de productividad Malmquist del período $\mathrm{t}$ orientado a las salidas es

$$
M_{0}{ }^{t}\left(x^{t}, y^{t}, x^{t+1}, y^{t+1}\right)=D_{0}{ }^{t}\left(x^{t+1}, y^{t+1}\right) / D_{0}{ }^{t}\left(x^{t}, y^{t}\right)
$$


$M_{0} t\left(x^{t}, y^{t}, x^{t+1}, y^{t+1}\right) \operatorname{coteja}\left(x^{t+1}, y^{t+1}\right)$ a $\left(x^{t}, y^{t}\right)$ mediante la comparación de sus respectivas distancias para el período tecnología t. $\mathrm{Mo}^{t}\left(\mathrm{x}^{\mathrm{t}}, \mathrm{y}^{\mathrm{t}}, \mathrm{x}^{\mathrm{t}+1}, \mathrm{y}^{\mathrm{t}+1}\right)$ se basa en funciones de distancia de salida definidas en relación con una tecnología de referencia que satisface los retornos variables a escala. Esto permitió a Grifell y Lovell (1995) mostrar que $M_{0}{ }^{t}\left(x^{t}, y^{t}, x^{t+1}, y^{t+1}\right) \geq \leq 1$ según el crecimiento de la productividad, el estancamiento o la disminución de la productividad, el efecto neto de las economías de escala, se ha producido entre los períodos $\mathrm{t}$ y $\mathrm{t}+1$, desde la perspectiva del período $t$ de tecnología.

Un periodo orientado a las salidas $\mathrm{t}+1$ del índice de productividad de Malmquist se define de forma similar $M_{0}{ }^{t+1}\left(x^{t}, y^{t}, x^{t+1}, y^{t+1}\right)$, reemplazando el

$$
\begin{aligned}
& \operatorname{Moc}^{t}\left(x^{t}, y^{t}, x^{t+1}, y^{t+1}\right)=\operatorname{Doc}^{t}\left(x^{t+1}, y^{t+1}\right) / \operatorname{Doc}^{t}\left(x^{t}, y^{t}\right) \quad \text { [7] } \\
& =\left[D^{t}\left(x^{t+1}, y^{t+1}\right) / D_{0}^{t+1}\left(x^{t+1}, y^{t+1}\right)\right] \bullet\left[D_{0}^{t+1}\left(x^{t+1}, y^{t+1}\right) / D_{0}^{t}\left(x^{t}, y^{t}\right)\right]
\end{aligned}
$$

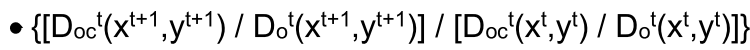

La descomposición planteada por Ray y Desli (1997) de $\operatorname{Moc}^{t}\left(x^{t}, \mathbf{y}^{t}, \mathbf{x}^{t+1}, \mathbf{y}^{t+1}\right)$ es:

El primer término en esta descomposición es un término de cambio tecnológico calculado en relación con los VRS, utilizando los datos del período $t+1$. El segundo término es un término de cambio de eficiencia relativa también calculado en relación con los VRS. El tercer término es un efecto de escala que mide el cambio en la eficiencia con relación al período $t$ de tecnología usando datos $\left(x^{t}, y^{t}\right)$ y $\left(x^{t+1}, y^{t+1}\right)$.

De tal manera que, en resumen, se tiene; si:

$M_{0}>1$ indica una mayor productividad en el período $t+1$ que en el período $t$.

$M_{0}>1$ significa una disminución de la productividad en el período $t+1$ en comparación con $\mathrm{t}$. 
$M_{0}=1$ implica un estancamiento en la productividad entre el período $\mathrm{t} y \mathrm{t}+1$.

El índice de Malmquist ha sido utilizado para evaluar los cambios en la productividad en diferentes escenarios, para los últimos años algunos autores como Örkcü et al. (2016) estudian 21 aeropuertos en Turquía del 2009 a 2014, Rácz y Vestergaard (2016) trabajan sobre las plantas de energía de biogás en Dinamarca, Martínez-Damián et al. (2013) evalúan la productividad en México, Aparicio et al. (2016) observan la brecha de rendimiento entre escuelas secundarias públicas y privadas dependientes del Gobierno en el País Vasco (España), Seijas e Iglesias (2013) estudian el cambio en la productividad de los Servicios Regionales de Salud españoles, Ferreira et al. (2016) analizan 145 aeropuertos de tres continentes, Cervantes y Martínez (2010) estudian las fincas cafetaleras de Veracruz y $\mathrm{Yu}$ et al. (2016) analizan la industria de la pulpa y el papel en China.

Ya en la parte financiera, Soltane (2014) investiga los cambios de productividad de 33 instituciones de microfinanzas de Oriente Medioy África del Norte durante el periodo 2006-2011, Galagedera et al. (2016) estudian los fondos mutuos familiares de los Estados Unidos de 1999 a 2008, Wijesiri y Meoli (2015) observan los cambios de productividad de 20 instituciones microfinancieras en Kenia. Específicamente para los bancos, Tortosa-Ausina et al. (2008) analizan los índices de productividad de las cajas de ahorros españolas en el periodo 1992-1998 y Kao y Liu, 2016 estudian los bancos comerciales taiwaneses desde el 2008 al 2013.

Para el caso colombiano, no se encuentra ninguna publicación cuyo análisis se centre en la medición de la productividad mediante el índice de Malmquist, para los bancos tanto nacionales como extranjeros.

\section{DISEÑO ESPECÍFICO}

$\begin{array}{lll}\text { La } & \text { Superintendencia } & \text { Financiera } \\ \text { de } & \text { Colombia (2017) } & \text { es la }\end{array}$ fuente de información.

Delimitación de las DMU: dado que se estudia el sector bancario colombiano, las DMU son los bancos que año tras año, y desde el 2002 y hasta el 2016, reportaron sus estados financieros a la Superintendencia Financiera de Colombia; se conformó un grupo de 43 bancos, teniendo en cuenta todos los bancos existentes en el periodo de estudio. Durante este periodo cinco bancos del Gobierno reportaron a la SFC; pero, por ser del Gobierno, se retiraron de la base de datos acogiendo lo expresado por Charnes et al. (1985) en cuanto a que instituciones del Gobierno solo son comparables entre sí. Adicionalmente, un banco privado que fue liquidado en 1999 también reportó sus estados financieros, pero por no ser comparable, 
porque no estaba en pleno funcionamiento, también se suprimió de la base de datos. En la Tabla 1 se muestra el número de bancos que para cada año hacen parte de la investigación.

Tabla 1. Número de bancos que hacen parte del estudio

\begin{tabular}{cc}
\hline Año & Bancos \\
2002 & 24 \\
2003 & 24 \\
2004 & 24 \\
2005 & 19 \\
2006 & 15 \\
2007 & 15 \\
2008 & 17 \\
2009 & 17 \\
2010 & 18 \\
2011 & 22 \\
2012 & 22 \\
2013 & 23 \\
2014 & 21 \\
2015 & 24 \\
2016 & 24 \\
\hline
\end{tabular}

Fuente: elaboración propia.

Delimitación de entradas y salidas: dado que DEA es un modelo no paramétrico de frontera, en el cual no es necesario establecer previamente la función de producción, y que las variables determinantes del modelo son los recursos utilizados (entradas) y lo que se obtiene del proceso de transformación de ellos (salidas), las variables utilizadas en esta investigación se muestran en la Tabla 2.
Modelo DEA específico: índice de productividad de Malmquist, descomposición realizada por Ray y Desly. Planteado mediante las ecuaciones [7] y [8].

Tabla 2. Variables de entrada y de salida

\begin{tabular}{cc}
\hline Entradas & Salidas \\
\hline Activo corriente & Ingresos operacionales \\
Propiedades & Utilidad neta \\
planta y equipo & \\
Pasivo no corriente & \\
Patrimonio & \\
\hline
\end{tabular}

Fuente: elaboración propia

\section{RESULTADOS}

En la Tabla 3 están los resultados para todos los 37 bancos que hacen parte del estudio. Los bancos que han cambiado de nombre a lo largo del periodo de estudio aparecen con el último nombre.

En el $64 \%$ de los periodos la productividad promedio mejoró (IM>1), siendo el periodo 2003-2004 el que muestra un mejor resultado; tiempo en el cual se considera que el sector bancario colombiano estuvo en pleno desarrollo y consolidación. En promedio, esta mejora en la productividad se debe al componente del cambio tecnológico calculado en relación con los VRS (Figura 1). Los bancos que más contribuyeron a la obtención de estos resultados fueron: Santander Colombia y GNB Sudameris. 
Tabla 3. Índice de Malmquist del año 2002 al año 2016

\begin{tabular}{|c|c|c|c|c|c|c|c|c|c|c|c|c|c|c|}
\hline $\begin{array}{l}\text { Índice de Malmquist } \\
\text { (IM \%) }\end{array}$ & 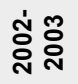 & ஜั่ & ठั่ & ம்่ ठั & ठั่े & 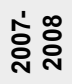 & ڤั่ & के̀ेंํํํ & ㅎํํ둥 & 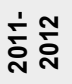 & ஸั่ & ஸें & 它 & กั่ \\
\hline BANCOS & IM & IM & IM & IM & IM & IM & IM & IM & IM & IM & IM & IM & IM & IM \\
\hline DE BOGOTÁ & 1,17 & 0,99 & 0,97 & 0,83 & 1,54 & 1,09 & 0,84 & 1,12 & 1,12 & 0,82 & 0,96 & 1,13 & 1,18 & 1,06 \\
\hline POPULAR & 1,32 & 1,03 & 1,21 & 0,81 & 1,23 & 1,06 & 1,01 & 0,8 & 0,97 & 1,02 & 0,89 & 0,94 & 0,75 & 0,96 \\
\hline CORPBANCA & & & & & & & & & & & 0,76 & 1,08 & 1,44 & 1,18 \\
\hline SANTANDER COLOMBIA & 0,71 & 3,34 & 0,45 & 1,04 & 1,09 & 1,03 & 1 & 1 & 1 & & & & & \\
\hline BANCOLOMBIA & 1,33 & 1,05 & 1,02 & 0,86 & 0,99 & 1,08 & 0,91 & 1,01 & 0,96 & 0,75 & 1,25 & 1,19 & 1,02 & 0,95 \\
\hline SCOTIABANK & & 1 & 008 & & 1 & 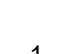 & 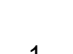 & & & 1 & & & & \\
\hline CITIBANK & 0,76 & 1,09 & 1 & 1 & 1,13 & 1,07 & 1 & 0,95 & 0,99 & 0,99 & 1,04 & 1 & 1 & 1 \\
\hline HSBC COLOMBIA & 1,02 & 1,17 & 1,44 & 0,63 & 1,88 & 1,1 & 0,84 & 0,89 & 1,05 & 1,02 & 0,68 & & & \\
\hline GNB SUDAME & 0,62 & 2,65 & 1,37 & 0,93 & 1,19 & 1,03 & 1,09 & 1,02 & 0,9 & 0,85 & 0,9 & 1,56 & 0,81 & 1,01 \\
\hline BBVA & 2,03 & 1,55 & 0,79 & 1,12 & 1,04 & 1,07 & 0,98 & 1 & 1,04 & 1 & 1,02 & 1,03 & 1,18 & 0,87 \\
\hline HELM BANK & 1,43 & 1,41 & 1,17 & 0,94 & 1,5 & 0,86 & 0,69 & 0,66 & 1,21 & 1,02 & 1 & & & \\
\hline UNIÓN COLOMBIA & 1,08 & 1,38 & 0,72 & & & & & & & & & & & \\
\hline OCCIDENTE & 1,11 & 1,11 & 0,99 & 0,96 & 1,16 & 1,21 & 0,71 & 0,92 & 1 & 0,93 & 0,94 & 2,07 & 0,78 & 0,97 \\
\hline $\begin{array}{l}\text { STANDARD } \\
\text { CHARTERED }\end{array}$ & 1 & 1 & & & & & & & & & & & & \\
\hline TEQUENL & 0,63 & 1,64 & & & & & & & & & & & & \\
\hline CAJA & 1,02 & 1,2 & 1,6 & 1,31 & 0,76 & 1,19 & 0,7 & 0,77 & 1,06 & 1,1 & 1,09 & 0,95 & 1,08 & 0,99 \\
\hline BANSUPERIOR & 0,99 & 1,11 & 0,57 & & & & & & & & & & & \\
\hline BANKBOS & 1 & 1 & & & & & & & & & & & & \\
\hline DAVIVIENDA & 1,47 & 1,16 & 1,19 & 0,72 & 1,06 & 1,41 & 0,64 & 0,83 & 0,9 & 1,02 & 1,04 & 1,22 & 1,03 & 1,12 \\
\hline & 2,24 & 1,15 & 1,12 & 1 & 1 & 1 & 1 & 1 & 1 & 1 & 1 & 1,01 & 1 & 1 \\
\hline MEGAB & 0,98 & 1,06 & 1,04 & & & & & & & & & & & \\
\hline GRANAHORRA & 1,43 & 1,29 & 1,12 & & & & & & & & & & & \\
\hline COLMENA & 1,68 & 1,2 & & & & & & & & & & & & \\
\hline CONAVI & 1,29 & 1,41 & & & & & & & & & & & & \\
\hline AV VILLAS & 1,36 & 1,26 & 1,39 & 0,69 & 1,7 & 0,76 & 1,02 & 0,9 & 0,97 & 0,98 & 1,03 & 1 & 1,12 & 0,85 \\
\hline PROCREDIT & & & & & & & 0,9 & 0,87 & 1,73 & 1,15 & 0,88 & 1,33 & 1,27 & 0,66 \\
\hline BANCAMÍA & & & & & & & 3,92 & 1,08 & 1 & 1 & 1 & 1 & 0,96 & 0,81 \\
\hline WWB & & & & & & & & & 0,93 & 1,17 & 1 & 1 & 1 & 1 \\
\hline COOMEVA & & & & & & & & & & 1,1 & 1 & 1 & 1,14 & 1 \\
\hline FINANDINA & & & & & & & & & & 1 & 1 & 1 & 1 & 1 \\
\hline FALABELLA & & & & & & & & & & 1,01 & 1 & 1 & 0,96 & 0,85 \\
\hline $\mathrm{PICHINCHA}$ & & & & & & & & & & 1,03 & 0,9 & 1,14 & 1,16 & 0,99 \\
\hline COOPCENTRAL & & & & & & & & & & & & 1,83 & 0,71 & 1,09 \\
\hline SANTANDER DE NE & cIOs & & & & & & & & & & & 2,16 & 1 & 1 \\
\hline MUNDO MUJER & & & & & & & & & & & & & & 1 \\
\hline MULTIBANK & & & & & & & & & & & & & & 0,6 \\
\hline BANCOMPARTIR & & & & & & & & & & & & & & 0,94 \\
\hline PROMEDIO & 1,2 & 1,34 & 1,06 & 0,92 & 1,22 & 1,06 & 1,07 & 0,91 & 1,08 & 1 & 0,97 & 1,22 & 1,03 & 0,95 \\
\hline
\end{tabular}

Fuente: elaboración propia 
En cuatro periodos existe disminución de la productividad promedio $(\mathrm{IM}<1)$, la mayor disminución ocurre en el periodo 2009-2010, durante la primera crisis financiera mundial de este siglo y este comportamiento se debió principalmente al componente del cambio tecnológico calculado en relación con los VRS, a pesar de que el componente del cambio promedio en la eficiencia con respecto al periodo 2009 de tecnología denotó un comportamiento creciente y de mejora en la productividad. Para el último lapso (2015-2016) también se evidencia disminución en el promedio de la productividad, este tiempo coincide con el estancamiento de la economía mundial.

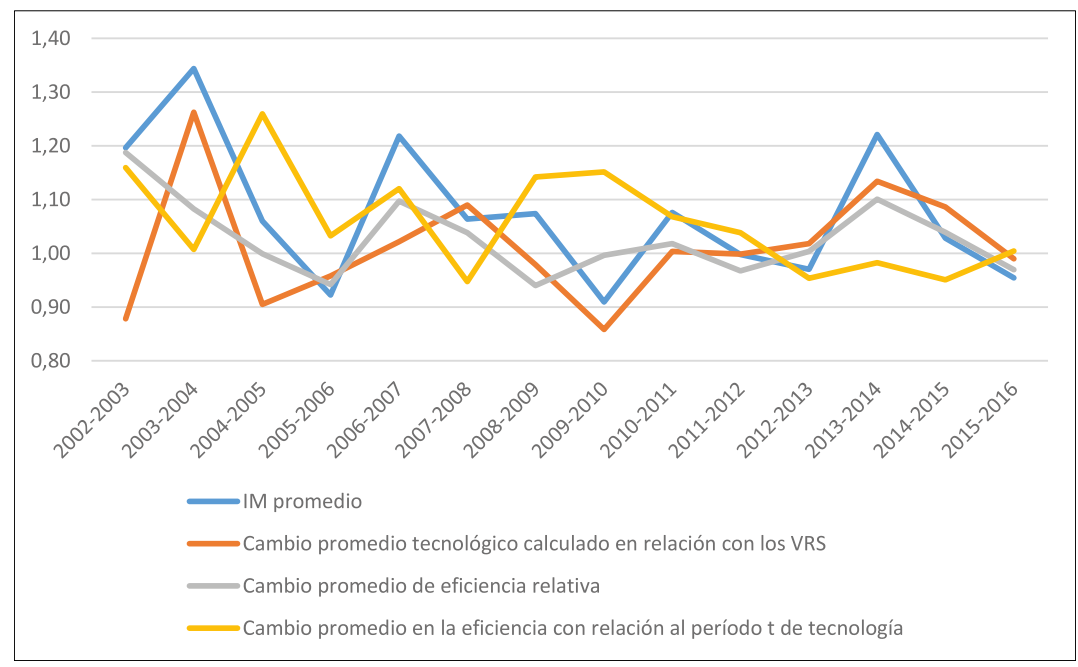

Figura 1. Comportamiento de los promedios.

Fuente: elaboración propia.

El $76 \%$ de todos los bancos del estudio tienen un IM promedio > 1, estos son los que tienen valores más altos: Colmena, Santander de Negocios, Conavi y Bancamía. Los tres primeros bancos le deben esto al componente del cambio promedio en la eficiencia relativa, mientras que para el último se debe al componente del cambio promedio en la eficiencia con relación al periodo $t$ de tecnología. En la Figura 2 se muestra el comportamiento del IM para estos bancos. 


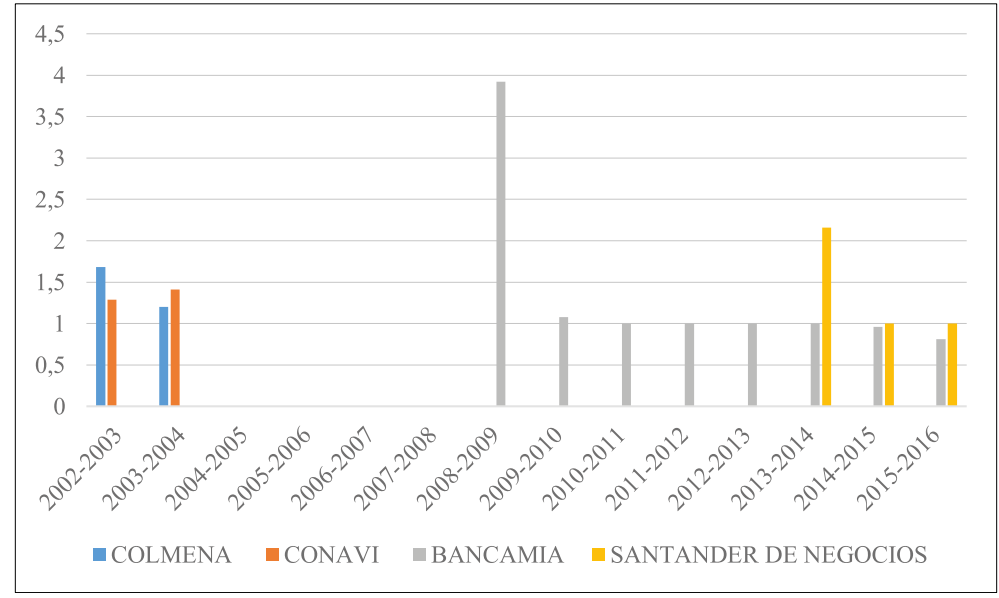

Figura 2. IM de los bancos, con IM promedio más alto.

Fuente: elaboración propia.

Los bancos que tienen el IM promedio < 1, son: Multibank, Compartir, Superior y Falabella; los dos primeros deben este comportamiento al cambio promedio de la eficiencia relativa, el Superior se lo debe prioritariamente al cambio promedio tecnológico calculado en relación con los VRS, y Falabella al cambio promedio en la eficiencia respecto al período t de tecnología. En la Figura 3 se muestra el comportamiento del IM para estos bancos.

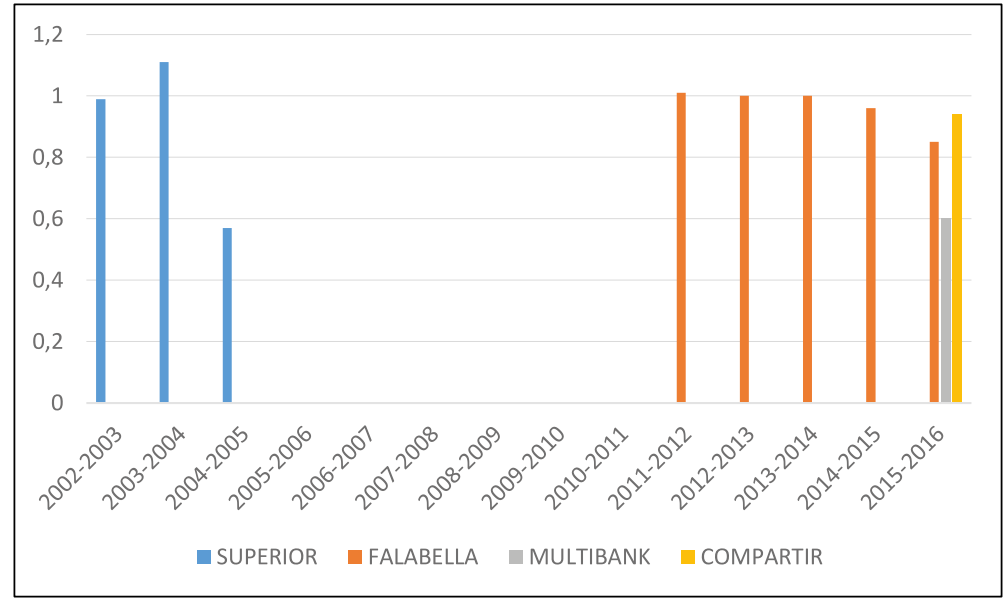

Figura 3. IM de los bancos con IM Promedio $<1$.

Fuente: elaboración propia. 
De los 24 bancos que reportaron información a la SFC para el último periodo 2015-2016, el $21 \%$ tiene un IM > 1 y para el $50 \%$ el IM es $<1$. Dentro del primer grupo destacan: Corpbanca y Davivienda; ambos tuvieron buenos resultados gracias a la mejora en el componente del cambio de la eficiencia relativa. Para el primer periodo 2002-
2003 también reportaron 24 bancos, pero en este tiempo el $67 \%$ tiene un $I M>1$, y el $25 \%<1$.

Ahora bien, para el IM en el último periodo y agrupando los bancos en nacionales y extranjeros se tienen los resultados que muestra la Tabla 4, en la cual queda claro que no existen diferencias ostensibles.

Tabla 4. Comportamiento del IM para el periodo 2015-2016

\begin{tabular}{cccc}
\hline Bancos & $\mathbf{I M}>\mathbf{1}$ & $\mathbf{I M}=\mathbf{1}$ & $\mathbf{I} \mathbf{M}<\mathbf{1}$ \\
\hline Nacionales & $21 \%$ & $4 \%$ & $50 \%$ \\
Extranjeros & $20 \%$ & $3 \%$ & $50 \%$ \\
\hline
\end{tabular}

Fuente: elaboración propia.

Pero otra es la situación cuando se analiza el comportamiento del IM promedio (Tabla 5), pues allí hay una diferencia de 8 puntos porcentuales a favor de los bancos extranjeros que muestran mejora en la productividad y una diferencia de 13 puntos porcentuales a favor de los bancos nacionales cuando el índice revela disminución en la productividad.

Tabla 5. Comportamiento del IM promedio para el periodo 2015-2016

\begin{tabular}{cccc}
\hline Bancos & IM P>1 & IM P=1 & IM P<1 \\
\hline Nacionales & $72 \%$ & $21 \%$ & $7 \%$ \\
Extranjeros & $80 \%$ & $0 \%$ & $20 \%$ \\
\hline
\end{tabular}

Fuente: elaboración propia

En cuanto a cómo impactan las adquisiciones al IM, se presentan resultados disimiles; por ejemplo, con resultados positivos en los cuales el $\mathrm{IM}>1$ está el caso de Corpbanca, que adquirió el Banco Helm; GNB Sudameris, que adquirió el Banco HSBC; el BBVA, que compró Granahorrar; y el Banco Caja Social, que compró Colmena. Para el Banco Colpatria, que obtuvo el
Scotiabank, no hubo cambio en la productividad $(\mathrm{IM}=1)$. Mientras que en los siguientes casos la productividad disminuyó $(\mathrm{IM}<1)$ : Davivienda, al adquirir el Banco Superior; el Banco de Occidente, al comprar el Unión Colombiano; Bancolombia, al comprar el Banco Conavi; GNB Sudameris, al obtener el Banco Tequendama; y el Banco Bogotá al comprar a Megabanco. Cuando 
las adquisiciones son de bancos del Gobierno, sucede lo mismo: Davivienda adquirió Bancafé y Granbanco y obtuvo un aumento en la productividad en cada caso. Pero cuando el Banco de Occidente compró el Banco Aliadas, su productividad disminuyó.
Ahora bien, analizando el comportamiento de los grupos financieros respecto al índice de Malmquist, se presenta la Figura 4, la cual evidencia que todos los grupos tienen IM promedio $>1$.

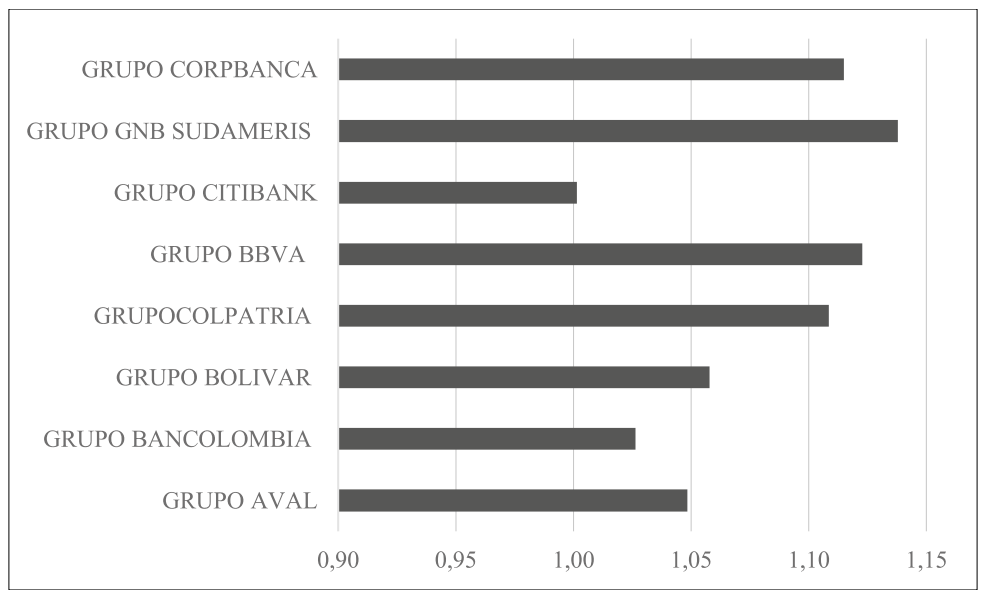

Figura 4. IM Promedio grupos.

Fuente: elaboración propia.

\section{CONCLUSIONES}

El sector financiero desempeña una función trascendental en toda economía, pues es el llamado a realizar la asignación y provisión de recursos monetarios. En Colombia la economía ha sido jalonada por este sector durante varios años; aún más, en época de desaceleración mundial económica es el que ha reportado altos niveles de ganancias, por ejemplo, para el año 2016 a pesar de la recesión mundial, este sector generó un incremento del $24.8 \%$ en sus ganancias.
El sector bancario colombiano surge de las crisis del siglo XX y se prepara para abordar el siglo XXI, desplegando una estrategia de conformación de grupos financieros de origen nacional. En los últimos 15 años, periodo de esta investigación, se ha dado un gran movimiento en cuanto al número de bancos en el sector en cada año. Aunque este periodo inició con 24 bancos en el año 2002, descontando los bancos propiedad del Gobierno, y cerró el 2016 con este mismo número, esto no quiere decir que no hayan existido movimientos dentro del periodo. 
Los bancos Conavi, Santander Colombia, Colmena, Scotiabank, Granahorrar, HSBC Colombia, Megabanco, Helm, Bansuperior, Unión colombiano y Tequendama desaparecieron del sector, porque fueron comprados o porque se fusionaron. El Banco Standard Chartered cerró voluntariamente y el Banco Bankboston liquidó también de manera voluntaria. Ingresaron los siguientes bancos: Bancompartir, Corpbanca, Multibank, Procredit Colombia, Bancamía, Mundo Mujer, WWB, Santander de Negocios, Coomeva, Coopcentral, Finandina, Pichincha y Falabella.

Dado que para aplicar la metodología DEA no es necesario predeterminar la función de producción y se puede trabajar un conjunto de multientradas y multisalidas para obtener un único indicador por DMU y por periodo de tiempo estudiado, se ajusta perfectamente al sector bancario.

En el $64 \%$ de los periodos la productividad promedio mejoró, siendo el periodo 2003-2004 el que muestra un mejor resultado; tiempo en el cual se considera que el sector bancario colombiano experimentó procesos de desarrollo y consolidación. Al componente del cambio tecnológico calculado en relación con los VRS, es al que se le debe este crecimiento. En cuatro periodos se evidenció disminución de productivi- dad, la mayor disminución ocurrió en el periodo 2009-2010, coincidente con la primera crisis financiera mundial de este siglo; para el último periodo también se evidenció disminución, en este se revela igualmente estancamiento de la economía mundial.

El $76 \%$ de los bancos tienen un IM promedio $>1$, los bancos que tienen el IM promedio $<1$, son el $11 \%$.

Para el primer periodo de estudio, el $67 \%$ de los bancos tienen el IM > 1, y el $25 \%<1$. Mientras que para el último periodo es el $21 \%$ y el $50 \%$ respectivamente.

Se demuestra que las adquisiciones tienen un impacto impredecible en el IM.

Ahora bien, analizando el comportamiento de los grupos financieros respecto al índice de Malmquist, se establece que para todos el IM promedio es $>1$. Es necesario evidenciar el comportamiento interno del Grupo Aval, pues es el que tiene más de un banco (Figura 5); para concluir que en el periodo 2005-2006 todos los bancos tuvieron disminución de la productividad y que para el último periodo solo el Banco de Bogotá obtuvo mejora.

Adicionalmente, en la Figura 6 se muestra el comportamiento del IM de los grupos para los 14 periodos de estudio. 


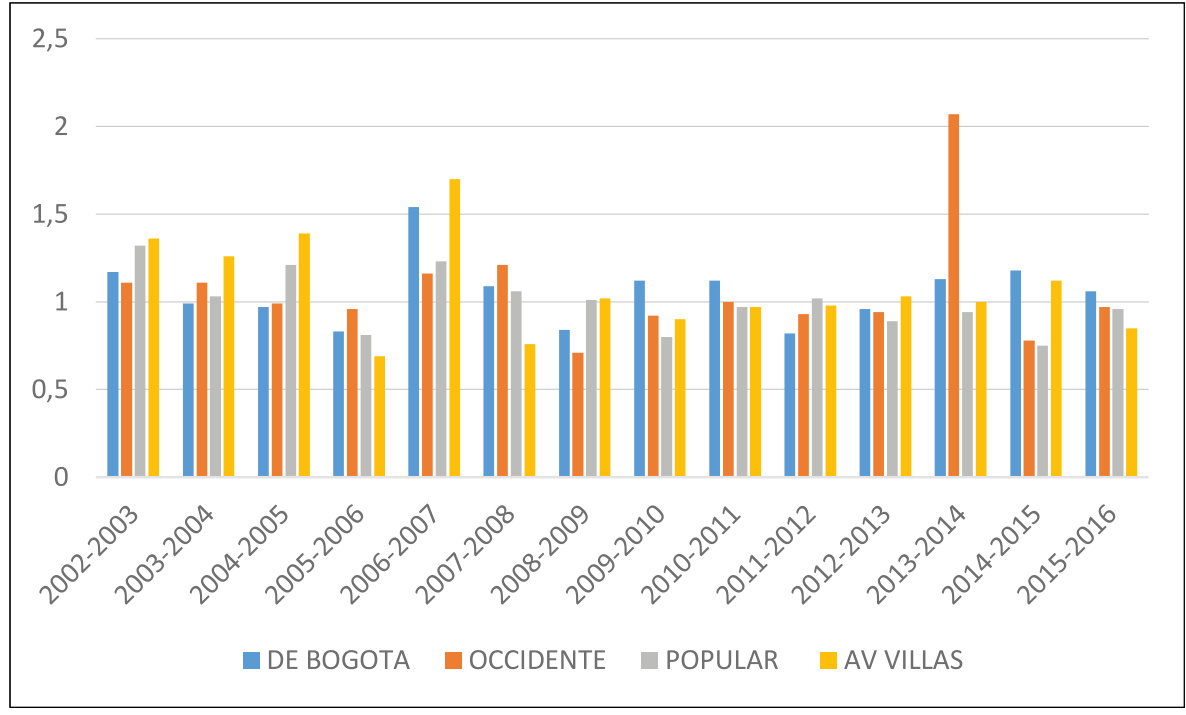

Figura 5. IM de los bancos del Grupo Aval.

Fuente: elaboración propia.

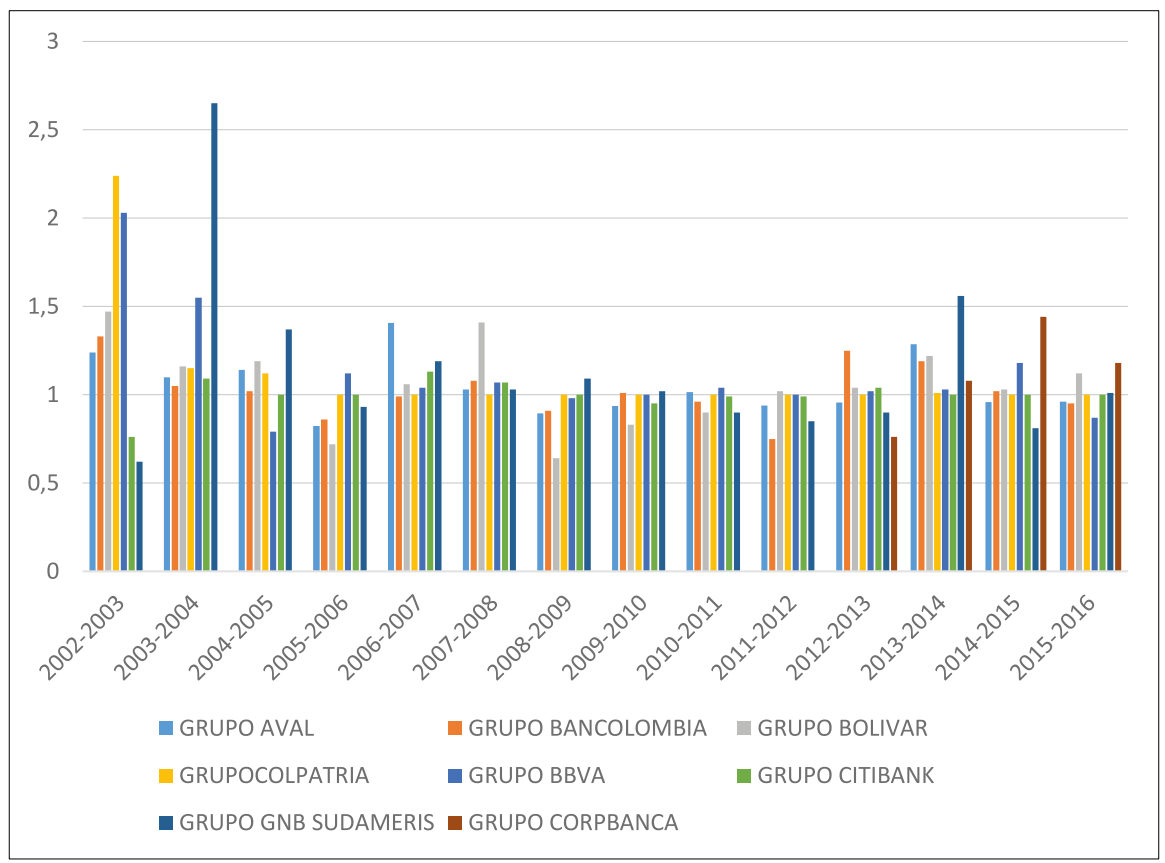

Figura 6. IM de los grupos del sector bancario colombiano.

Fuente: elaboración propia. 


\section{AGRADECIMIENTOS}

A los evaluadores anónimos de la Revista, por sus comentarios y sugerencias.

DECLARACIÓN DE CONFLICTOS

\section{DE INTERESES}

La autora declara que no existe conflicto de intereses.

\section{FINANCIAMIENTO}

Este trabajo no tuvo ningún tipo de financiamiento institucional. 


\section{REFERENCIAS}

[1] Aparicio, J., Crespo-Cebada, E., Pedraja-Chaparro, F., \& Santín, D. (2016). Comparing School Ownership Performance Using A Pseudo-Panel Database: A Malmquist-Type Index Approach. European Journal of Operational Research, 256, 533-542. https://doi.org/10.1016/j.ejor.2016.06.030

[2] Arango, M. (2006). Evolución y crisis del sistema financiero colombiano. Serie estudios y perspectivas. CEPAL.

[3] Asobancaria. (2017). Informe de tipificación de la banca colombiana en 2016. Asobancaria.

[4] Banco Davivienda. (2017). Informe de gestión 2016. Davivienda.

[5] Banco GNB Sudameris. (2017, 25 ago.). Historia. https://www.citibank.com.co/ institucional/historia.htm\#

[6] Barajas, A., Salazar, N., \& Steiner, R. (1999). Interest Spreads in Banking in Colombia, 1974-96. IMF Staff Papers, 46(2).

[7] BBVA. (25 de agosto de 2017). Banco Bilvao Vizcaya Argentaria Colombia. https:// www.bbva.com.co/meta/historia/

[8] Beck, T., Demirguç-Kunt, A., \& Levine, R. (2007). Financial, Inequality and the Poor. Journal of Economic Growth 12(1), 27-49. https://doi.org/10.1007/ s10887-007-9010-6

[9] Berger, A., Demirguç-Kunt, A., Levine, R., \& Haubrich, J. (2004). Bank Concentration and Competition: An Evolution in the Making. Journal of Money, Credit, and Banking, 36(3), 434-450. https://doi.org/10.1353/mcb.2004.0040

[10] BID. (2004). Progreso económico y social en América Latina. Informe 2005. El Ateneo.

[11] Caves, D., Christensen, L., \& Diewert, W. (1982). The Economic Theory of Index Numbers and Meaurement of Input, Output and Productivity. Econometrica, 50(6), 1393-1414. https://doi.org/10.2307/1913388

[12] Cervantes, O. \& Martínez, M. (2010). Eficiencia en las fincas cafetetaleras de Veracruz: índice de productividad de Malmquist. Rev. Mex. de Ec. y Agríc. y de los Rec. Nat. 3(1), 153-163. 
[13] Charnes, A., Cooper, W., Golany, B., Seiford, L., \& Stutz, J. (1885). Foundations of Data Envelopment Analysis and Pareto-Koopmans Empirical Production Functions. Journal of Econometrics, 30, 91-107. https://doi.org/10.1016/0304-4076(85)90133-2

[14] Cihák, M., Demirguç-Kunt, A., Feyen, E., \& Levine, R. (2012). Benchmarking Financial Systems around the World. World Bank. https://doi. org/10.1596/1813-9450-6175

[15] Citibank. (2017, 25 ago.). Citi España. http://www.citibank.com/spain/citi/historia. htm

[16] Citibank Colombia. (2017, 25 ago.). Citivalores. Quiénes somos. https://www. citibank.com.co/Citivalores/quienes_somos/index.htm

[17] Citigroup. (2017, 25 ago.). Citi institucional. https://www.citibank.com.co/institucional/historia.htm\#

[18] Claessens, S., \& Djankov, S. (1999). Ownership Concentration and Coporate Performance in The Czech Republic", University of Michigan Business School Working Paper, No. 227. Michigan Business School.

[19] Colpatria. (2017, 25 ago.). Colpatria Multibanca. https://www.colpatria.com/ Acerca-de/banco-colpatria/informacion-institucional/nuestra-organizacion

[20] Coltefinanciera. (2017, 20 ago.). Educación financiera. http://www. coltefinanciera.com.co/educacion-financiera/sistema-financiero/385-como-esta-estructurado-el-sistema-financiero-en-colombia

[21] Corpbanca. (2015). Presentación institucional. Corpbanca.

[22] Cuartas, A., Rodríguez, M., \& Rodríguez, N. (2012). Banking Institutions in Colombia, Consequence of A Constant Movement in Banking Sector. Revista MBA EAFIT, 32-53.

[23] Daude, C., \& Pascal, J. (2015). Efficiency and Contestability in the Colombian Banking System, OECD Economics Department Working Papers, No. 1203. OECD Publishing.

[24] Davivienda. (25 deagosto de 2017).Banco Davivienda.Quiénes somos. https://www. davivienda.com/wps/portal/inversionistasespanol/inversionistas/AcercaBanco/ quienes_somos/davivienda/!ut/p/b1/04_SjzQzszCysDAzMdWP0I_KSyzLTE8syczPS8wB8aPM4sNMDQK9vN0NDfw9PdwMPF18QkzMAwONvEMMgQoigQoMcABHA0L6w_Wj8CpxNYAqwGOFn0d-bqp-blSOpaeuoyIAOw 
[25] Dotoi, M., Epicoco, N., Falagario, M., \& Sciancalepore, F. (2015). A Cross-Efficency Fuzzy Data Envelopment Analysis Technique for Performance Evaluation of Decision Making Units Under Uncertainty. Computers \& Industrial Engineering, 79, 103-114. https://doi.org/10.1016/j.cie.2014.10.026

[26] Fare, R., Grosskopf, S., Lindgren, B., \& Ross, P. (1992). Productivity Changes in Swedish Pharmacies 1980-1989: A Non-Parametric Malmquist Approach. Journal of Productivity Analysis, 3, 85-102. https://doi.org/10.1007/BF00158770

[27] Fare, R., Grosskopf, S., Norris, M., \& Zhang, Z. (1994). Producticity Growrth Technical Progress, and Efficiency Change in Industrialized Countries. The American Economic Review, 84(1), 66-83.

[28] Ferreira, D., Marques, R., \& Pedro, M. (2016). Comparing Efficiency of Holding Business Model and Individual Management Model of Airports. Journal of Air Transport Management, 57, 168-183. https://doi.org/10.1016/j. jairtraman.2016.07.020

[29] Fogafín. (2017, 27 ago.). Qué es Fogafin? https://www.fogafin.gov.co/Default/ que-es-fogafin/quienes-somos

[30] Galagedera, U., Watson, J., Premachandra, I., \& Chen, Y. (2016). Modeling Leakage in Two-Stage DEA Models: An Application to US Mutual Fund Families. Omega 61, 62-77. https://doi.org/10.1016/j.omega.2015.07.007

[31] García, N., Grifoni, A., López, J., \& Mejía, D. (2013). La educación financiera en América Latina y el Caribe. Situación actual y perspectivas. Serie Políticas Públicas y Transformación Productiva $N^{\circ} 12$. CAF.

[32] Ghasemi, M., Ignatius, J., Lozano, S., Emrouznejad, A., \& Hatami-Marbini, A. (2015). A Fuzzy Expected Value Approach Under Generalized Data Envelopment Analysis. Knowledge-Based Systems, 89, 148-159. https://doi.org/10.1016/j. knosys.2015.06.025

[33] Gómez-Gonzáles, J., \& Kiefer, N. (2007, 25 ago.). Bank Failure: Evidence From The Colombian Financial Crisis, OCC Economics Working Paper. https://www. occ.gov/publications/publications-by-type/occ-working-papers/2008-2000/ wp2007-2.pdf

[34] Grifell, E., \& Lovell, C. (1995). A Note on the Malmquist Productivity Index. Economics Letters 47, 169-175. https://doi.org/10.1016/0165-1765(94)00497-P 
[35] Grifell-Tatjé, E., \& Lovell, C. (1999). A Generalized Malmquist Productivity Index. Sociedad de Estadóstica e Investigación Operativa, 7(1), 81-101. https://doi. org/10.1007/BF02564713

[36] Grupo Aval. (2017, 25 ago.). Nuestras compañias. https://www.grupoaval.com/ wps/portal/grupo-aval/aval/nuestras-companias

[37] Grupo Bancolombia. (2017, 25 ago.). Grupo Bancolombia. Quiénes somos. https://www.grupobancolombia.com/wps/portal/acerca-de/informacion-corpora tiva/quienes-somos/

[38] Grupo Sura. (2017, 25 ago.). Grupo Sura. Inversiones. https://www.gruposura. com/Inversiones/grupo-bancolombia/Paginas/default.aspx

[39] IDB. (2004). Unlocking Credit: The Quest for Deep and Stable Bank Lending, Economic and Social Progress in Latin America Series. Inter-American Development Bank.

[40] Iyer, K., \& Banerjee, P. (2016). Measuring and Benchmarking Managerial Efficiency of Project Execution Schedule Performance. International Journal of Project Management, 34(2), 219-236. https://doi.org/10.1016/j.ijproman.2015.10.008

[41] Kao, C., \& Liu, S. (2016). A Parallel Production Frontiers Approach for Intertemporal Efficiency Analysis: The Case of Taiwanese Commercial Banks. European Journal of Operational Research, 255, 411-421. https://doi.org/10.1016/j. ejor.2016.04.047

[42] Lau, K. (2013). Measuring Distribution Efficiency of a Retail Network through Data Envelopmnet Analysis. International Journal Production Economics, 146(2), 598-611. https://doi.org/10.1016/j.ijpe.2013.08.008

[43] Martínez-Damiàn, M., Brambila-Paz, J., \& García-Mata, R. (2013). Índice de Malmquist y productividad estatal en México. Agricultura, Sociedad y Desarrollo, 10(3), 359-369.

[44] Meisel, A. (1990). El Banco de la República: antecedentes, evolución y estructura. Banco de la República.

[45] Örkcü, H., Dogan, M., \& Genc, A. (2016). An Evaluation of the Oprational Efficiency of Turkish Airports Using Data Envelopmente Analysis and the Malmquist Productivity Index: 2009-2014 Case. Transport Policy, 48, 92-104. https://doi.org/10.1016/j.tranpol.2016.02.008 
[46] Rácz, V., \& Vestergaard, N. (2016). Productivity and Efficiency Measurement of the Danish Centralized Biogas Power Sector. Renewable Energy, 92, 397-404. https://doi.org/10.1016/j.renene.2016.02.024

[47] Ray, S., \& Desli, E. (1997). Productivity Growth, Technical Progress and Efficiency Change in Industrialized Countries: Comment. American Economic Review 87(5), 1033-1039.

[48] Rodríguez, G. (2011). Indicadores DEA (Data Envelopment Analysis) de eficiencia y productividad para las actividades de extensión universitaria. Aplicación en la Universidad Nacional de Colombia. Centro Editorial Facultad de Ciencias Económicas Universidad Nacional de Colombia.

[49] Secretaría del Senado. (2017, 20 ago.). Leyes desde 1992, vigencia expresa y control de constitucionalidad. http://www.secretariasenado.gov.co/senado/basedoc/ estatuto_organico_sistema_financiero.html

[50] Seijas, A., \& Iglesias, G. (2013). Evolución de la productividad y asociación con la satisfacción en la atención hospitalaria y especializada de los sistemas sanitarios de las Comunidades Autónomas. Investigaciones Regionales, 27, 7-32.

[51] Shepard, R. (1970). The Theory of Cost and Production Functions. Princeton University Press.

[52] Singh, A., Belaisch, A., Collyns, C., De Masi, P., Krieger, R., Meredith, G., \& Rennhack, R. (2005). Stabilization and Reform in Latin America: A Macroeconomic Perspective on the Experience Since The Early 1990s. International Monetary Fund. https://doi.org/10.5089/9781589062504.084

[53] Soltane, B. (2014). Total Factor Productivity Change Of MENA Microfinance Institutions: A Malmquist Productivity Index Approach. Economic Modelling, 39, 182-189. https://doi.org/10.1016/j.econmod.2014.02.035

[54] Superintendencia Financiera de Colombia -SFC-. (2017, 20 ago.). Superintendencia Financiera de Colombia. https://www.superfinanciera.gov.co/jsp/loader.jsf?1Servi cio $=$ Publicaciones\&lTipo $=$ publicaciones\&lFuncion=loadContenidoPublicacion \&id $=10083710$

[55] Tortosa-Ausina, E., Grifell-Tatjé, E., Armero, C., \& David, C. (2008). Sensitivity Analysis of Efficiency and Malmquist Productivity Indices: An Application to Spanish Savings Banks. European Journal of Operational Research 184, 1062 1084. https://doi.org/10.1016/j.ejor.2006.11.035 
[56] Uribe, J. (2013). Nota Editorial: El sistema financiero colombiano: estructura y evolución reciente. Revista del Banco de la República, 86(1023), 5-17.

[57] Wei, Q., \& Yang, H. (2010). A Data Envelopment Analysis (DEA) Evaluation Method Based on Sample Decision Making Units. International Journal of Information Technology \& Decision Making, 9(4), 601-624. https://doi.org/10.1142/ S021962201000397X

[58] Wijesiri, M., \& Meoli, M. (2015). Productivity Change of Microfinance Institutions in Kenya: Abootstrap Malmquist Approach. Journal of Retailing and Consumer Services, 25, 115-121. https://doi.org/10.1016/j.jretconser.2015.04.004

[59] Yang, M., Li, Y., Chen, Y., \& Liang, L. (2014). An Equilibrium Efficiency Frontier Data Envelopment Analysis Approach for Evaluating Decision-Making Units With Fixed-Sum Outputs. European Journal of Operational Research, 239(2), 479-489. https://doi.org/10.1016/j.ejor.2014.05.013

[60] Yu, C., Shi, L., Wang, Y., Chang, Y., \& Cheng, B. (2016). The Eco-Efficiency of Pulp and Paper Industry in China: An Assessed Based on Slacks-Based Measure and Melmquist E Luemberger Index. Journal of Cleaner Production, 127, 511-521. https://doi.org/10.1016/j.jclepro.2016.03.153 
